\title{
A Novel Application of Solids Characterization by Environmental Scanning Electron Microscopy (ESEM) Utilizing a Peltier Stage
}

\author{
R.J. Maxwell* and J.A. Hanko** \\ *Trace Analysis, Pharmacia Corporation, 7000 Portage Road, Kalamazoo, MI 49001 \\ **Global Pharmaceutical Sciences, Pharmacia Corporation, 4901 Searle Parkway Skokie, Il 60077
}

The advent of the Environmental Scanning Electron Microscope (ESEM) has brought about new opportunities to utilize this technique to characterize pharmaceutically active compounds. The ability to examine materials under a partial pressure of water vapor allows for imaging an uncoated specimen. This flexibility allows for the routine nondestructive examination of a variety of materials that could not be achieved with a coated specimen (e.g., hydrated and/or solvated crystal lattices. By utilizing an uncoated specimen, dynamic imaging of the surface during dehydration and or desolvation can now be routinely observed. With the addition of a Peltier stage, the specimen can be exposed to a wide range of relative humidity $(\mathrm{RH})$ conditions by varying the stage temperature and/or pressure of the gas within the environmental chamber.

Using this technique for in situ monitoring a pharmaceutical sample environment, specifically a solid dosage form, was of considerable interest to understand the affects of humidity on sample integrity. Given the typical tablet size, the Peltier stage sample capacity, and the concern about a suitable cross-section to represent the bulk sample, a fundamental question arose: "Is the effective RH at the analysis site on a tablet surface the same as at the tablet/Peltier contact site for a given temperature/pressure combination?" Scheme 1 summarizes this question. The high degree of temperature control provided by the Peltier stage coupled with ESEM pressure controls, allows the operator to span the entire $\mathrm{RH}$ scale and maintain conditions for prolonged periods of time. However, the instrumental data output for temperature/pressure/RH conditions are only accurate within the Peltier stage environment. For situations where samples extend beyond the Peltier stage, a method for determining RH at the site of analysis is useful.

Thus a quick and sensitive technique for estimating the actual temperature/RH at the analysis site is critical for a better understanding of observed specimen effects. This was achieved by placing a few salt grains (see Figure 1A for established deliquescence conditions) on the elevated surface of a compressed tablet and recording the deliquesce point. By recording the output conditions at which deliquesce was achieved and factoring in the known deliquescence conditions for the salt, the temperature/RH at the analysis site (see Figure 1B) can be calculated. 


\section{Scheme 1:}


Figure 1: (A) $\mathrm{NaCl}$ crystals on the Peltier Stage starting to deliquesce. (9.9Torr/ $\left.15^{\circ} \mathrm{C} / 77 \% \mathrm{RH}\right)$. (B) View of $\mathrm{NaCl}$ crystals on Surface of a Tablet at 10.2Torr $/ 15^{\circ} \mathrm{C}$ corresponding to $80 \% \mathrm{RH}$ at the sample/Peltier contact point 